\title{
Wonham filter with random parameters: Rate of convergence and error bounds
}

\author{
X. Guo* G. Yin ${ }^{\dagger}$
}

July 29,2003

\begin{abstract}
Let $\alpha(t)$ be a finite-state continuous-time Markov chain with generator $Q=\left(q^{i j}\right) \in$ $\mathbb{R}^{m \times m}$ and state space $\mathcal{M}=\left\{z^{1}, \ldots, z^{m}\right\}$. When the state space and the generator are known a priori, the best estimator of $\alpha(t)$ (in terms of mean square error) under noisy observation is the celebrated Wonham filter. This paper addresses the estimation issue when values of the state space or values of the generator are unknown a priori. In each case, we propose a (suboptimal) filter and prove its convergence to the desired Wonham filter under simple conditions. Moreover, we obtain the rate of convergence via the study of both the mean square and the higher moment error bounds.
\end{abstract}

Key words: Wonham filter, Rate of convergence, Approximation, Error bounds.

${ }^{*}$ School of ORIE, Cornell University, Ithaca, NY 14853. Email: xinguo@orie.cornell.edu.

${ }^{\dagger}$ Department of Mathematics, Wayne State University, Detroit, MI 48202. Supported in part by the National Science Foundation under grant DMS-0304928. Email: gyin@math.wayne.edu. 


\section{Introduction}

Given a probability space $(\Omega, \mathcal{F}, P)$ and $t \in[0, T]$ for some $T>0$. Suppose that $\alpha(t)$ is a finite-state continuous time Markov process with state space $\mathcal{M}=\left\{z^{1}, \ldots, z^{m}\right\}$ and generator $Q=\left(q^{i j}\right) \in \mathbb{R}^{m \times m}$, so that the transition probabilities are

$$
P^{i j}(h)=P\left(\alpha(t+h)=z^{j} \mid \alpha(t)=z^{i}\right),
$$

and

$$
P^{i j}(h)=\left\{\begin{array}{lll}
1-q^{i} h+o(h), & i=j, & h \rightarrow 0 \\
q^{i j} h+o(h), & i \neq j, & h \rightarrow 0,
\end{array}\right.
$$

where

$$
q^{i}=\sum_{i \neq j} q^{i j} .
$$

Let us further assume that the Markov process $\alpha(t)$ is observed with the observation process $y(t)$ such that

$$
\left\{\begin{array}{l}
d y(t)=\alpha(t) d t+\sigma(t) d w(t), \\
y(0)=0 \text { w.p. } 1,
\end{array}\right.
$$

where $w(\cdot)$ is a standard 1-dimensional Brownian motion that is independent of $\alpha(t)$, and $\sigma(\cdot): \mathbb{R} \mapsto \mathbb{R}$, with $\sigma(t) \geq c$ for all $t \in[0, T]$ and some $c>0$, is a continuously differentiable function. Note that (3) indicates that the basic observation model has the form of "signal plus noise", and that the distribution of $y(t)$ is non-Gaussian but is a Gaussian mixture due to the jump processes $\alpha(t)$.

In this framework, one of the classical results known as the Wonham filter concerns estimating $\alpha(t)$ based on the observation $y(\cdot)$. When the values of the states $z^{1}, \cdots, z^{m}$ and the generator $Q$ are known a priori and fixed, the Wonham filter [9] provides the optimal filter in the sense of mean square error. It was the first finite-dimensional filter for non-Gaussian processes, and remains as one of the very few known finite-dimensional filters to-date. (The first rigorous development of non-linear filters for diffusion-type processes was [5]. For a more detailed treatment of filtering problems, see [7, Vol 1 \& 2]; for general references on Markov processes and stochastic differential equations, see $[1,2,8]$; for some recent references on filtering and estimation, see $[10,11]$.)

It is natural to take a step further and consider the estimation problem when there are additional uncertainties in the value of the state space $z^{1}, \ldots, z^{m}$ or of the generator $\left(q^{i j}\right)$. For instance, one may consider the case when $z^{i}$ 's are unknown a priori, and their values 
are known only up to a certain distribution. This problem arises frequently in for example, Bayesian statistics, where priors could be drawn from a distribution. One possible solution is to apply a Monte-Carlo approach which entails the replacement of states in the Wonham filter by their simulated or approximated values. Such approach raises important questions such as the rate of convergence and error estimates. To some extent, many of these problems may be viewed as a robustness issues - this is the central topic of this paper.

Our results. Our first main result is the construction of approximating filters when only noisy or simulated values $z^{i}$ or $\left(q^{i j}\right)$ are available. We then prove that these suboptimal filters converge to the desired Wonham filter under simple ergodicity conditions.

We also evaluate the accuracy of the approximations and derive the approximation error bounds. These bounds, including both mean square (or $L_{2}$ bounds) as well as bounds based on higher moments, provide results on the rate of convergence and enable one to assess the quality of the approximations.

Organization. Section 2 contains the main results. Proofs of the main theorems are in Section 3. Section 4 concludes with some remarks.

\section{Main results}

\subsection{Wonham filter}

Let $\alpha(t)$ be a finite-state continuous time Markov process with state space $\mathcal{M}=\left\{z^{1}, \ldots, z^{m}\right\}$ and generator $Q=\left(q^{i j}\right) \in \mathbb{R}^{m \times m}$, as defined earlier.

Given (3), define

$$
p(t)=\left(p^{1}(t), \ldots, p^{m}(t)\right) \in \mathbb{R}^{1 \times m},
$$

with

$$
\begin{aligned}
& p^{i}(t)=P\left(\alpha(t)=z^{i} \mid y(s), 0 \leq s \leq t\right), i=1, \ldots, m, \\
& p^{i}(0)=p_{0}^{i} .
\end{aligned}
$$

It was proved in [9] that this conditional density (posterior probability) provides the minimal mean square error, and satisfies the following system of stochastic differential equations

$$
\begin{aligned}
d p^{i}(t)=\sum_{j=1}^{m} & p^{j}(t) q^{j i} d t-\sigma^{-2}(t) \bar{\alpha}(t)\left[z^{i}-\bar{\alpha}(t)\right] p^{i}(t) d t \\
& +\sigma^{-2}(t)\left[z^{i}-\bar{\alpha}(t)\right] p^{i}(t) d y(t), i=1, \ldots, m
\end{aligned}
$$


where

$$
\bar{\alpha}(t)=\langle p(t), z\rangle \stackrel{\text { def }}{=} \sum_{i=1}^{m} z^{i} p^{i}(t), \quad z=\left(z^{1}, \ldots, z^{m}\right)^{\prime},
$$

and $v^{\prime}$ denotes the transpose of $v$.

Adopting a vector notation, define

$$
A(t) \stackrel{\text { def }}{=} \operatorname{diag}\left(z^{1}-\bar{\alpha}(t), \ldots, z^{m}-\bar{\alpha}(t)\right)=\left(\begin{array}{lll}
z^{1}-\bar{\alpha}(t) & & \\
& \ddots & \\
& & z^{m}-\bar{\alpha}(t)
\end{array}\right) .
$$

Then the Wonham filter can be rewritten as

$$
\left\{\begin{array}{l}
d p(t)=p(t) Q d t-\sigma^{-2}(t) \bar{\alpha}(t) p(t) A(t) d t+\sigma^{-2}(t) p(t) A(t) d y(t) \\
p(0)=p_{0}
\end{array}\right.
$$

\subsection{Approximate Wonham filter using $\left\{\widehat{z}_{n}\right\}$}

Now, let us assume that $z^{i}$ 's are not available, and that only their noise corrupted measurements or observations or distributional information are at our disposal. We assume further that $\left(q^{i j}\right)$ remains unchanged and known a priori.

In particular, we assume that a sequence of observations of the form

$$
\widehat{z}_{n}=\left(\widehat{z}_{n}^{1}, \ldots, \widehat{z}_{n}^{m}\right)^{\prime} \in \mathbb{R}^{m \times 1} \text { such that } E \widehat{z}_{n}=z
$$

can be obtained. For example, $\widehat{z}_{n}=z+\xi_{n}$, where $\left\{\xi_{n}\right\}$ is a sequence of $\mathbb{R}^{m}$-valued zero mean observation noise satisfying appropriate conditions. We proceed to construct the approximate filter.

First, define

$$
\bar{z}_{n}=\frac{1}{n} \sum_{j=1}^{n} \widehat{z}_{j}
$$

Then in lieu of (6), we have a sequence of approximations $p_{n}(t)$ given by

$$
\left\{\begin{array}{l}
d p_{n}(t)=p_{n}(t) Q d t-\sigma^{-2}(t) \bar{\alpha}_{n}(t) p_{n}(t) A_{n}(t) d t+\sigma^{-2}(t) p_{n}(t) A_{n}(t) d y(t) \\
p_{n}(0)=p_{0}
\end{array}\right.
$$

where

$$
\bar{\alpha}_{n}(t)=\left\langle p_{n}(t), \bar{z}_{n}\right\rangle, \quad A_{n}(t)=\operatorname{diag}\left(\bar{z}_{n}^{1}-\bar{\alpha}_{n}(t), \ldots, \bar{z}_{n}^{m}-\bar{\alpha}_{n}(t)\right)
$$


Since $\left\{p_{n}(t)\right\}$ is a sequence of approximations of the posterior density $p(t)$, we may appropriately normalize it to ensure its boundedness [9].

Define

$$
e_{n}(t)=p_{n}(t)-p(t)
$$

Then, $e_{n}(t)$ satisfies

$$
\left\{\begin{aligned}
d e_{n}(t)=e_{n}(t) Q d t & -\sigma^{-2}(t)\left[\bar{\alpha}_{n}(t)-\bar{\alpha}(t)\right] p_{n}(t) A_{n}(t) d t \\
& -\sigma^{-2}(t) \bar{\alpha}(t) e_{n}(t) A_{n}(t) d t \\
& -\sigma^{-2}(t) \bar{\alpha}(t) p(t)\left[A_{n}(t)-A(t)\right] d t \\
& +\sigma^{-2}(t) e_{n}(t) A_{n}(t) d y(t) \\
& +\sigma^{-2}(t) p(t)\left[A_{n}(t)-A(t)\right] d y(t), \\
e_{n}(0)=0 . &
\end{aligned}\right.
$$

To obtain the desired limit result, we impose the following conditions.

(A1) $\left\{\widehat{z}_{n}\right\}$ is a stationary ergodic sequence that satisfies $E \widehat{z}_{n}=z$ and is uniformly bounded. The sequence $\left\{\widehat{z}_{n}\right\}$ is independent of the Markov chain $\alpha(\cdot)$ and the Brownian motion $w(\cdot)$.

Remark 1. The uniform boundedness of the sequence $\left\{\widehat{z}_{n}\right\}$ is not a restriction since one may use, for example, truncated normal random distributions. It conveniently allows $p_{n}(t)$ to have approximated posterior probability vector interpretation. Although no independence is assumed for the sequence $\left\{\widehat{z}_{n}\right\}$, in simulation one often uses i.i.d. sequences for simplicity. Ergodicity implies that $\bar{z}_{n} \rightarrow z$ w.p.1. The conditions cover a large class of processes. Examples of interests include the case when $\left\{\widehat{z}_{n}\right\}$ is a stationary $\phi$-mixing sequence, which in turn is ergodic (see [4, pp. 488-489]). That is, $\bar{z}_{n} \rightarrow z$ w.p.1. In addition, if the mixing measure $\phi_{n}$ satisfies $\sum_{n} \phi_{n}^{1 / 2}<\infty$, then $\sqrt{n}\left(\bar{z}_{n}-z\right)$ converges in distribution to a normal random variable with mean 0 and covariance

$$
\Xi=E \widehat{z}_{0} \widehat{z}_{0}^{\prime}+\sum_{k=1}^{\infty} E \widehat{z}_{0} \widehat{z}_{k}^{\prime}+\sum_{k=1}^{\infty} E \widehat{z}_{k} \widehat{z}_{0}^{\prime} .
$$

This ascertains the convergence and rate of convergence of the sequence $\bar{z}_{n}$.

Theorem 2. Under (A1),

$$
\sup _{0 \leq t \leq T} E\left|e_{n}(t)\right|^{2} \rightarrow 0 \quad \text { as } n \rightarrow \infty .
$$


It is well-known that convergence in $L_{2}$ implies convergence in probability. Thus, the following is immediate.

Corollary 3. Under (A1), for any $\eta>0$,

$$
\lim _{n \rightarrow \infty} P\left(\left|e_{n}(t)\right| \geq \eta\right)=0
$$

Next, define $e_{n}^{\kappa}(t)=n^{\kappa} e_{n}(t)$ for any $0<\kappa \leq 1 / 2$. Then the following estimates hold.

Theorem 4. Under (A1),

$$
\sup _{0 \leq t \leq T} E\left|e_{n}^{\kappa}(t)\right|^{2}=\left\{\begin{array}{ll}
o(1), & 0<\kappa<1 / 2, \\
O(1), & \kappa=1 / 2,
\end{array} \text { as } n \rightarrow \infty .\right.
$$

Finally, we have error bounds for higher moments.

Theorem 5. Assume (A1).

(i) For any positive integer $\ell>1$,

$$
\sup _{0 \leq t \leq T} E\left|e_{n}^{\kappa}\right|^{2 \ell}=\left\{\begin{array}{ll}
o(1), & 0<\kappa<1 / 2, \\
O(1), & \kappa=1 / 2,
\end{array} \text { as } n \rightarrow \infty .\right.
$$

(ii) For $\kappa=1 / 2$, denote $\widetilde{e}_{n}(t)=e_{n}^{1 / 2}(t)$. Then

$$
\sup _{0 \leq t \leq T} E \exp \left(\left|\widetilde{e}_{n}(t)\right|\right)=O(1), \text { as } n \rightarrow \infty \text {. }
$$

\subsection{Approximate Wonham filter using $\left\{\widehat{Q}_{n}\right\}$}

Now, we assume uncertainty in $\left(q^{i j}\right)$ with its sequence of noise corrupted observations $\widehat{Q}_{n}=$ $\left(\widehat{q}_{n}^{i j}\right) \in \mathbb{R}^{m \times m}$. As in the previous section, the approximate filter can be built accordingly by defining

$$
\bar{Q}_{n}=\frac{1}{n} \sum_{l=1}^{n} \widehat{Q}_{l}
$$

and by redefining a sequence of approximations $p_{n}(t)$ with

$$
\left\{\begin{array}{l}
d p_{n}(t)=p_{n}(t) \bar{Q}_{n} d t-\sigma^{-2}(t) \bar{\alpha}_{n}(t) p_{n}(t) A_{n}(t) d t+\sigma^{-2}(t) p_{n}(t) A_{n}(t) d y(t) \\
p_{n}(0)=p_{0}
\end{array}\right.
$$


where

$$
\bar{\alpha}_{n}(t)=\left\langle p_{n}(t), z\right\rangle, \quad A_{n}(t)=\operatorname{diag}\left(z^{1}-\bar{\alpha}_{n}(t), \ldots, z^{m}-\bar{\alpha}_{n}(t)\right) .
$$

Let $e_{n}(t) \stackrel{\text { def }}{=} p_{n}(t)-p(t)$. Then

$$
\left\{\begin{aligned}
d e_{n}(t)=e_{n}(t) \bar{Q}_{n} d t & +p(t)\left(\bar{Q}_{n}-Q\right) d t \\
& -\sigma^{-2}(t)\left[\bar{\alpha}_{n}(t)-\bar{\alpha}(t)\right] p_{n}(t) A_{n}(t) d t \\
& -\sigma^{-2}(t) \bar{\alpha}(t) e_{n}(t) A_{n}(t) d t \\
& -\sigma^{-2}(t) \bar{\alpha}(t) p(t)\left[A_{n}(t)-A(t)\right] d t \\
& +\sigma^{-2}(t) e_{n}(t) A_{n}(t) d y(t) \\
& +\sigma^{-2}(t) p(t)\left[A_{n}(t)-A(t)\right] d y(t), \\
e_{n}(0)=0 . &
\end{aligned}\right.
$$

(A2) $\left\{\widehat{Q}_{n}\right\}$ is a stationary ergodic sequence that satisfies $E \widehat{Q}_{n}=Q$ and is uniformly bounded. The sequence $\left\{\widehat{Q}_{n}\right\}$ is independent of the Markov chain $\alpha(\cdot)$ and the Brownian motion $w(\cdot)$.

In a similar fashion, we can derive the following results:

Theorem 6. Assume (A2). Then

$$
\sup _{0 \leq t \leq T} E\left|e_{n}(t)\right|^{2} \rightarrow 0 \text { as } n \rightarrow \infty
$$

Remark 7. For ease of exposition, the assumption (A2) is stronger than needed. For instance, Theorem 6 holds when the uniform boundedness of $\left\{\widehat{Q}_{n}\right\}$ is replaced by $E\left|\widehat{Q}_{n}\right|^{2}<$ $\infty$. This remark applies to the following Theorems as well when the uniform boundedness is replaced by appropriate higher moment conditions.

Theorem 8. Assume (A2).

(i) For any positive integer $\ell>1$,

$$
\sup _{0 \leq t \leq T} E\left|e_{n}^{\kappa}(t)\right|^{2 \ell}=\left\{\begin{array}{ll}
o(1), & 0<\kappa<1 / 2, \\
O(1), & \kappa=1 / 2,
\end{array} \text { as } n \rightarrow \infty .\right.
$$

(ii) For $\kappa=1 / 2$, denote $\widetilde{e}_{n}(t)=e_{n}^{1 / 2}(t)$. Then

$$
\sup _{0 \leq t \leq T} E \exp \left(\left|\widetilde{e}_{n}(t)\right|\right)=O(1), \text { as } n \rightarrow \infty \text {. }
$$


Remark 9. Theorems 4 and 8 provide a rate of convergence result. It asserts that the convergence speed is of the order $n^{1 / 2}$. For any $\kappa<1 / 2$, a trivial limit is obtained. Note that the convergence is in the sense of mean square convergence. Thus, Theorem 4 and Theorem 8 tell us how the mean square error depends on the size of the sample.

Finally, the same techniques can be used to build Wonham filters using both $\left\{\widehat{z}_{n}\right\}$ and $\left\{\widehat{Q}_{n}\right\}$. Formally, the approximate filters so designed have the same form as (13). Assuming (A1), (A2), and the independence of $\left\{\widehat{z}_{n}\right\}$ and $\left\{\widehat{Q}_{n}\right\}$, then the conclusions of Theorem 8 continue to hold.

\section{Proofs}

\subsection{Notation}

Throughout the rest of the paper, we use the notation $K, K_{T}, K_{T, \ell}$ to denote some generic positive constants, in which the subscripts highlight their dependence on the indicated quantities. These constants are generic in the sense their values may be different for different usage. That is, the convention $K+K=K$ and $K K=K$ is used. Before going through the proofs, here are some basic inequalities used throughout.

\subsection{Review of some inequalities}

Lemma 10. Suppose that $f(t)$ is $\mathcal{F}_{t}$-measurable and that it satisfies $\int_{0}^{T} E|f(t)|^{2} d t<\infty$, where $\mathcal{F}_{t}$ is the $\sigma$-algebra generated by $\{w(s), \alpha(s): s \leq t\}$. Then

$$
E\left|\int_{0}^{t} f(s) d y(s)\right|^{2} \leq K_{T} \int_{0}^{t} E|f(s)|^{2} d s,
$$

where $y(s)$ is given by $(3)$.

Proof: It is straightforward that

$$
\begin{aligned}
E\left|\int_{0}^{t} f(s) d y(s)\right|^{2} & =E\left|\int_{0}^{t} f(s)[\alpha(s) d s+\sigma(s) d w(s)]\right|^{2} \\
& \leq K E\left|\int_{0}^{t} f(s) \alpha(s) d s\right|^{2}+K E\left|\int_{0}^{t} f(s) \sigma(s) d w(s)\right|^{2} \\
& \leq K E \int_{0}^{t}|f(s)|^{2} d s+K \int_{0}^{t} E|f(s) \sigma(s)|^{2} d s \\
& \leq K_{T} \int_{0}^{t} E|f(s)|^{2} d s
\end{aligned}
$$

The lemma follows. 
Lemma 11 (Gronwall's inequality). Let $u(t)$ and $g(t)$ be non-negative continuous functions on $[0, T]$ for which the inequality

$$
u(t) \leq C+\int_{0}^{t} g(s) u(s) d s, \quad t \in[0, T]
$$

holds, where $C$ is a non-negative constant. Then

$$
u(t) \leq C \exp \left(\int_{0}^{t} g(s) d s\right), \quad t \in[0, T] .
$$

Proof: See $[3$, p. 36$]$.

Lemma 12 For any real numbers $J_{1}, J_{2}, J_{3}, J_{4}, J_{5}$, and $r \geq 1$,

$$
\left|J_{1}+J_{2}+J_{3}+J_{4}+J_{5}\right|^{r} \leq K_{r}\left(\left|J_{1}\right|^{r}+\left|J_{2}\right|^{r}+\left|J_{3}\right|^{r}+\left|J_{4}\right|^{r}+\left|J_{5}\right|^{r}\right),
$$

where $K_{r}$ is a positive constant depending on $r$.

Proof: This is obtained via repeated applications of the following inequality: For any real numbers $a, b$, and $r \geq 1$,

$$
|a+b|^{r} \leq 2^{r-1}\left(|a|^{r}+|b|^{r}\right) .
$$

\subsection{Proof of Theorem 2}

Writing (9) in a variational form and noting $e_{n}(0)=0$, we obtain

$$
\begin{aligned}
e_{n}(t)= & -\int_{0}^{t} \sigma^{-2}(s)\left[\bar{\alpha}_{n}(s)-\bar{\alpha}(s)\right] p_{n}(s) A_{n}(s) e^{Q(t-s)} d s \\
& -\int_{0}^{t} \sigma^{-2}(s) \bar{\alpha}(s) e_{n}(s) A_{n}(s) e^{Q(t-s)} d s \\
& -\int_{0}^{t} \sigma^{-2}(s) \bar{\alpha}(s) p(s)\left[A_{n}(s)-A(s)\right] e^{Q(t-s)} d s \\
& +\int_{0}^{t} \sigma^{-2}(s) e_{n}(s) A_{n}(s) e^{Q(t-s)} d y(s) \\
& +\int_{0}^{t} \sigma^{-2}(s) p(s)\left[A_{n}(s)-A(s)\right] e^{Q(t-s)} d y(s) .
\end{aligned}
$$

For a suitable function $g(\cdot)\left(\right.$ e.g., $\mathcal{F}_{t}$-measurable and $\left.\int_{0}^{T} E|g(t)|^{2} d t<\infty\right)$, by the CauchySchwarz inequality,

$$
E\left|\int_{0}^{t} g(s) d s\right|^{2} \leq\left(\int_{0}^{t} d s\right)\left(\int_{0}^{t} E|g(s)|^{2} d s\right) \leq K_{T} \int_{0}^{t} E|g(s)|^{2} d s .
$$


Thus, we arrive at

$$
\begin{aligned}
E\left|e_{n}(t)\right|^{2} \leq K_{T}\{ & \int_{0}^{t} E\left|\sigma^{-2}(s)\right|^{2}\left|\bar{\alpha}_{n}(s)-\bar{\alpha}(s)\right|^{2}\left|p_{n}(s)\right|^{2}\left|A_{n}(s)\right|^{2}\left|e^{Q(t-s)}\right|^{2} d s \\
& +\int_{0}^{t} E\left|\sigma^{-2}(s)\right|^{2}|\bar{\alpha}(s)|^{2}\left|e_{n}(s)\right|^{2}\left|A_{n}(s)\right|^{2}\left|e^{Q(t-s)}\right|^{2} d s \\
& +\int_{0}^{t} E\left|\sigma^{-2}(s)\right|^{2}|\bar{\alpha}(s)|^{2}|p(s)|^{2}\left|A_{n}(s)-A(s)\right|^{2}\left|e^{Q(t-s)}\right|^{2} d s \\
& +\int_{0}^{t} E\left|\sigma^{-2}(s)\right|^{2}\left|e_{n}(s)\right|^{2}\left|A_{n}(s)\right|^{2}\left|e^{Q(t-s)}\right|^{2} d s \\
& \left.+\int_{0}^{t} E\left|\sigma^{-2}(s)\right|^{2}|p(s)|^{2}\left|A_{n}(s)-A(s)\right|^{2}\left|e^{Q(t-s)}\right|^{2} d s\right\} .
\end{aligned}
$$

Since we are working with the finite horizon $[0, T]$, the uniform boundedness of $\sigma^{-2}(s), p_{n}(s)$, $A_{n}(s)$, and $e^{Q(t-s)}$ on $0 \leq s \leq t \leq T$ then implies

$$
E\left|e_{n}(t)\right|^{2} \leq K_{T}\left\{\int_{0}^{t} E\left|\bar{\alpha}_{n}(s)-\bar{\alpha}(s)\right|^{2} d s+\int_{0}^{t} E\left|A_{n}(s)-A(s)\right|^{2} d s+\int_{0}^{t} E\left|e_{n}(s)\right|^{2} d s\right\} .
$$

Indeed, note that

$$
\bar{\alpha}_{n}(s)-\bar{\alpha}(s)=\left\langle p_{n}(s)-p(s), \bar{z}_{n}\right\rangle+\left\langle p(s), \bar{z}_{n}-z\right\rangle .
$$

Thus

$$
\int_{0}^{t} E\left|\bar{\alpha}_{n}(s)-\bar{\alpha}(s)\right|^{2} d s \leq K_{T}\left\{\int_{0}^{t} E\left|e_{n}(s)\right|^{2} d s+\int_{0}^{t} E\left|\bar{z}_{n}-z\right|^{2} d s\right\}
$$

Likewise,

$$
\int_{0}^{t} E\left|\bar{A}_{n}(s)-A(s)\right|^{2} d s \leq K_{T}\left\{\int_{0}^{t} E\left|e_{n}(s)\right|^{2} d s+\int_{0}^{t} E\left|\bar{z}_{n}-z\right|^{2} d s\right\}
$$

Substituting (20) and (21) into (19), by the Gronwall's inequality,

$$
E\left|e_{n}(t)\right|^{2} \leq K_{T} \delta_{n} \exp \left(K_{T} t\right) \leq K_{T} \delta_{n} \exp \left(K_{T} T\right)
$$

where

$$
\delta_{n}=\int_{0}^{T} E\left|\bar{z}_{n}-z\right|^{2} \rightarrow 0 \text { as } n \rightarrow \infty .
$$

Recall that $K_{T}$ is a generic positive constant depending on $T$. We have

$$
\sup _{0 \leq t \leq T} E\left|e_{n}(t)\right|^{2} \leq K_{T} \delta_{n} \rightarrow 0 \text { as } n \rightarrow \infty .
$$

The desired result then follows. 


\subsection{Proof of Theorem 5}

\subsubsection{Part (i)}

Using (16) and Lemma 12, we obtain that for a positive integer $\ell$ with $r=2 \ell>2$,

$$
E\left|e_{n}^{\kappa}(t)\right|^{2 \ell} \leq K_{\ell}\left(E\left|J_{1}\right|^{2 \ell}+E\left|J_{2}\right|^{2 \ell}+E\left|J_{3}\right|^{2 \ell}+E\left|J_{4}\right|^{2 \ell}+E\left|J_{5}\right|^{2 \ell}\right),
$$

where

$$
\begin{aligned}
& J_{1}=\int_{0}^{t} n^{\kappa} \sigma^{-2}(s)\left[\bar{\alpha}_{n}(s)-\bar{\alpha}(s)\right] p_{n}(s) A_{n}(s) e^{Q(t-s)} d s \\
& J_{2}=\int_{0}^{t} \sigma^{-2}(s) \bar{\alpha}(s) e_{n}^{\kappa}(s) A_{n}(s) e^{Q(t-s)} d s \\
& J_{3}=\int_{0}^{t} n^{\kappa} \sigma^{-2}(s) \bar{\alpha}(s) p(s)\left[A_{n}(s)-A(s)\right] e^{Q(t-s)} d s \\
& J_{4}=\int_{0}^{t} n^{\kappa} \sigma^{-2}(s) e_{n}(s) A_{n}(s) e^{Q(t-s)} d y(s) \\
& J_{5}=\int_{0}^{t} n^{\kappa} \sigma^{-2}(s) p(s)\left[A_{n}(s)-A(s)\right] e^{Q(t-s)} d y(s) .
\end{aligned}
$$

Similar to the estimates for $E\left|e_{n}^{\kappa}(t)\right|^{2}$ and in particular to (17), we have, from the Hölder's inequality,

$$
\begin{aligned}
E\left|\int_{0}^{t} g(s) d s\right|^{2 \ell} & \leq\left[\left(\int_{0}^{t} d s\right)^{1 /(1-(1 /(2 \ell)))}\left(\int_{0}^{t} E|g(s)|^{2 \ell}\right)^{1 /(2 \ell)}\right]^{2 \ell} \\
& \leq K_{T, \ell} \int_{0}^{t} E|g(s)|^{2 \ell} d s
\end{aligned}
$$

where $K_{T, \ell}>0$ is a generic positive constant depending on $T$ and $\ell$.

This inequality, together with the fact that $\sigma^{-2}(\cdot), p_{n}(\cdot)$, and $A_{n}(\cdot)$ are uniformly bounded in the interval $[0, T]$, and that $e^{Q(t-s)}$ is bounded for $0 \leq s \leq t \leq T$, suggests

$$
\begin{aligned}
E\left|J_{1}\right|^{2 \ell} & \leq K_{T, \ell} \int_{0}^{t} n^{2 \kappa \ell} E\left|\sigma^{-2}(s)\right|^{2 \ell}\left|\bar{\alpha}_{n}(s)-\bar{\alpha}(s)\right|^{2 \ell}\left|p_{n}(s)\right|^{2 \ell}\left|A_{n}(s)\right|^{2 \ell} \mid e^{Q(t-s)} d s \\
& \leq K_{T, \ell} \int_{0}^{t} n^{2 \kappa \ell} E\left|\bar{\alpha}_{n}(s)-\bar{\alpha}(s)\right|^{2 \kappa \ell} d s .
\end{aligned}
$$

Likewise, we obtain

$$
\begin{aligned}
& E\left|J_{2}\right|^{2 \ell} \leq K_{T, \ell} \int_{0}^{t} E\left|e_{n}^{\kappa}(s)\right|^{2 \ell} d s \\
& E\left|J_{3}\right|^{2 \ell} \leq K_{T, \ell} \int_{0}^{t} n^{2 \kappa \ell} E\left|A_{n}(s)-A(s)\right|^{2 \ell} d s .
\end{aligned}
$$


Let $f(\cdot)$ be a function satisfying $\int_{0}^{T} E|f(t)|^{2 \ell} d t<\infty$. Similar to the proof of Lemma 10, with the use of [7, Vol I, p. 131, Lemma 4.12], we obtain

$$
E\left|\int_{0}^{t} f(s) d y(s)\right|^{2 \ell} \leq K_{T, \ell} \int_{0}^{t} E|f(s)|^{2 \ell} d s .
$$

Thus,

$$
\begin{aligned}
& E\left|J_{4}\right|^{2 \ell} \leq K_{T, \ell} \int_{0}^{t} E\left|e_{n}^{\kappa}(s)\right|^{2 \ell} d s . \\
& E\left|J_{5}\right|^{2 \ell} \leq K_{T, \ell} \int_{0}^{t} E\left|A_{n}(s)-A(s)\right|^{2 \ell} d s .
\end{aligned}
$$

Combining the estimates obtained thus far and separating $e_{n}^{\kappa}(s)$ and $\bar{z}_{n}-z$ as done in (20) and (21), we arrive at

$$
E\left|e_{n}^{\kappa}\right|^{2 \ell} \leq K_{T, \ell}\left\{\int_{0}^{t} n^{2 \kappa \ell} E\left|\bar{z}_{n}-z\right|^{2 \ell}+\int_{0}^{t} E\left|e_{n}^{\kappa}(s)\right|^{2 \ell} d s\right\} .
$$

Note that

$$
E\left|\bar{z}_{n}-z\right|^{2 \ell} \leq \frac{1}{n} \sum_{l=0}^{n} E\left|\widehat{z}_{l}-z\right|^{2 \ell} .
$$

An application of the Gronwall's inequality yields that

$$
E\left|e_{n}^{\kappa}(t)\right|^{2 \ell} \leq K_{T, \ell} \zeta_{n}^{\kappa} \exp \left(K_{T, \ell} T\right),
$$

and in addition,

$$
\sup _{0 \leq t \leq T} E\left|e_{n}^{\kappa}(t)\right|^{2 \ell} \leq K_{T, \ell} \zeta_{n}^{\kappa} \exp \left(K_{T, \ell} T\right)
$$

where

$$
\zeta_{n}^{\kappa}=\int_{0}^{T} n^{2 \kappa \ell} E\left|\bar{z}_{n}-z\right|^{2 \ell} d s .
$$

Therefore,

$$
\zeta_{n}^{\kappa}= \begin{cases}O(1), & \kappa=1 / 2 \\ o(1), & 0<\kappa<1 / 2\end{cases}
$$

\subsubsection{Part (ii)}

In part (i), we have shown that $\left|\widetilde{e}_{n}(t)\right|^{2 \ell}=O(1)$ and that the bound is uniform in $t \in[0, T]$. Similar to [6, p. 142], $E\left|\widetilde{e}_{n}(t)\right|^{2 \ell-1} \leq E^{(2 \ell-1) /(2 \ell)}\left|\widetilde{e}_{n}(t)\right|^{2 \ell}=O(1)$ uniformly in $t \in[0, T]$. In view of (23), we can make $K_{T, \ell} \leq K_{T}^{2 \ell}$. Thus

$$
E \exp \left(\left|\widetilde{e}_{n}(t)\right|\right)=\sum_{l=0}^{\infty} \frac{E\left|\widetilde{e}_{n}(t)\right|^{l}}{l !} \leq K \sum_{l=0}^{\infty} \frac{K_{T}^{l}}{l !}<\infty .
$$

The proof is concluded. 


\subsection{Proof of Theorem 4}

Similar to (19), we obtain

$$
\begin{aligned}
E\left|e_{n}^{\kappa}(t)\right|^{2} \leq K_{T}\left\{\int_{0}^{t} E\left(n^{2 \kappa}\left|\bar{\alpha}_{n}(s)-\bar{\alpha}(s)\right|^{2}\right) d s\right. & +\int_{0}^{t} E\left(n^{2 \kappa}\left|A_{n}(s)-A(s)\right|^{2}\right) d s \\
& \left.+\int_{0}^{t} E\left|e_{n}^{\kappa}(s)\right|^{2} d s\right\} .
\end{aligned}
$$

Note that by virtue of Remark 1,

$$
\begin{aligned}
& E\left(n^{2 \kappa}\left|\bar{\alpha}_{n}(t)-\alpha(t)\right|^{2}\right)= \begin{cases}o(1), & 0<\kappa<1 / 2, \\
O(1), & \kappa=1 / 2,\end{cases} \\
& E\left(n^{2 \kappa}\left|A_{n}(t)-A(t)\right|^{2}\right)= \begin{cases}o(1), & 0<\kappa<1 / 2, \\
O(1), & \kappa=1 / 2,\end{cases}
\end{aligned}
$$

and the above bounds hold uniformly in $t \in[0, T]$. The rest of the proof is similar to the proof of previous theorems.

\section{$4 \quad$ Further remarks}

An approximation algorithm has been developed in this work. For simplicity, it was setup as a scalar problem. Extensions to vector-valued problems are straightforward. For example, one may consider the observation of the form

$$
d y(t)=g(\alpha(t)) d t+\sigma(t) d w(t), y(0)=0 \quad \text { w.p.1, }
$$

where $y(t) \in \mathbb{R}^{r}, g(\cdot): \mathcal{M} \mapsto \mathbb{R}^{r}, \sigma(\cdot): \mathbb{R}^{r \times r} \mapsto \mathbb{R}^{r}, w(\cdot)$ is standard $r$-dimensional Brownian motion, $\alpha(t)$ is a continuous-time Markov chain taking values in $\mathcal{M}=\{1, \ldots, m\}$. Then one can proceed with the corresponding Wonham filter and approximation when the state values are observed with noise. The results can be carried over with few modifications.

Acknowledgments The authors thank Amir Dembo for stimulating discussions and references.

\section{References}

[1] J. L. Doob, Stochastic Processes, Wiley Classic Library Edition, Wiley, New York, 1990.

[2] I.I. Gihman and A.V. Skorohod, Stochastic Differential Equations, Springer-Verlag, Berlin, 1972. 
[3] J. K. Hale, Ordinary Differential Equations, R.E. Krieger Publishing Co., 2nd Ed., Malabar, 1980.

[4] S. Karlin and H. M. Taylor, A First Course in Stochastic Processes, 2nd Edition, Academic Press, New York, NY, 1975.

[5] H.J. Kushner, On the differential equations satisfied by conditional probability densities of Markov processes with applications, SIAM J. Control, 2 (1964), 106-119.

[6] H. J. Kushner and G. Yin, Stochastic Approximation Algorithms and Applications, 2nd Ed., Springer-Verlag, New York, NY, 2003.

[7] R. S. Liptser and A. N. Shiryayev, Statistics of Random Processes I 83 II, SpringerVerlag, New York, NY, 2001.

[8] A. V. Skorohod, Studies in the Theory of Random Processes, Dover, New York, 1982.

[9] W.M. Wonham, Some applications of stochastic differential equations to optimal nonlinear filtering, SIAM J. Control, 2 (1965), 347-369.

[10] G. Yin and S. Dey, Weak convergence of hybrid filtering problems involving nearly completely decomposable hidden Markov chains, SIAM J. Control Optim., 41 (2003), $1820-1842$.

[11] O. Zeitouni and A. Dembo, Exact filters for the estimation of the number of transitions of finite-state continuous-time Markov processes, IEEE Trans. Information Theory, $\mathbf{3 4}$ (1988), 890-893. 